\title{
THE CLOSEST KNOWN FLYBY OF A STAR TO THE SOLAR SYSTEM
}

\author{
Eric E. MamajeK ${ }^{1}$, Scott A. Barenfeld ${ }^{2}$, Valentin D. Ivanov ${ }^{3,4}$, Alexei Y. Kniazev ${ }^{5,6,7}$, Petri Väisänen ${ }^{5,6}$, \\ YURI BELETSKY ${ }^{8}$, AND HENRI M. J. BOFFIN ${ }^{3}$ \\ ${ }^{1}$ Department of Physics \& Astronomy, University of Rochester, Rochester, NY 14627, USA; emamajek@pas.rochester.edu \\ 2 Department of Astronomy, California Institute of Technology, Pasadena, CA 91125, USA \\ ${ }^{3}$ European Southern Observatory, Av. Alonso de Cordova 3107, 19001 Casilla, Santiago 19, Chile \\ ${ }^{4}$ European Southern Observatory, Karl-Schwarzschild-Str. 2, D-85748 Garching bei München, Germany \\ ${ }^{5}$ South African Astronomical Observatory, P.O. Box 9, 7935 Observatory, Cape Town, South Africa \\ ${ }^{6}$ Southern African Large Telescope Foundation, P.O. Box 9, 7935 Observatory, Cape Town, South Africa \\ ${ }^{7}$ Sternberg Astronomical Institute, Lomonosov Moscow State University, Moscow, Russia \\ ${ }^{8}$ Las Campanas Observatory, Carnegie Institution of Washington, Colina el Pino, Casilla 601 La Serena, Chile \\ Received 2014 December 13; accepted 2015 January 18; published 2015 February 12
}

\begin{abstract}
Passing stars can perturb the Oort Cloud, triggering comet showers and potentially extinction events on Earth. We combine velocity measurements for the recently discovered, nearby, low-mass binary system WISE J072003.20084651.2 ("Scholz's star") to calculate its past trajectory. Integrating the Galactic orbits of this $\sim 0.15 M_{\odot}$ binary system and the Sun, we find that the binary passed within only $52_{-14}^{+23} \mathrm{kAU}\left(0.25_{-0.07}^{+0.11} \mathrm{pc}\right)$ of the Sun $70_{-10}^{+15} \mathrm{kya}(1 \sigma$ uncertainties), i.e., within the outer Oort Cloud. This is the closest known encounter of a star to our solar system with a well-constrained distance and velocity. Previous work suggests that flybys within $0.25 \mathrm{pc}$ occur infrequently $\left(\sim 0.1 \mathrm{Myr}^{-1}\right)$. We show that given the low mass and high velocity of the binary system, the encounter was dynamically weak. Using the best available astrometry, our simulations suggest that the probability that the star penetrated the outer Oort Cloud is $\sim 98 \%$, but the probability of penetrating the dynamically active inner Oort Cloud ( $<20 \mathrm{kAU}$ ) is $\sim 10^{-4}$. While the flyby of this system likely caused negligible impact on the flux of longperiod comets, the recent discovery of this binary highlights that dynamically important Oort Cloud perturbers may be lurking among nearby stars.
\end{abstract}

Key words: Galaxy: kinematics and dynamics - Oort Cloud - stars: individual (WISE J072003.20-084651.2, Scholz's star, HIP 85605)

\section{INTRODUCTION}

Perturbations by passing stars on Oort cloud comets have previously been proposed as the source of long-period comets visiting the planetary region of the solar system (Oort 1950; Biermann et al. 1983; Weissman 1996; Rickman 2014), and possibly for generating Earth-crossing comets that produce biological extinction events (Davis et al. 1984). Approximately $30 \%$, of craters with diameters $<10 \mathrm{~km}$ on the Earth and Moon are likely due to long-period comets from the Oort Cloud (Weissman 1996). Periodic increases in the flux of Oort cloud comets due to a hypothetical substellar companion have been proposed (Whitmire \& Jackson 1984); however, recent time series analysis of terrestrial impact craters are inconsistent with periodic variations (Bailer-Jones 2011), and sensitive infrared sky surveys have yielded no evidence for any wide-separation substellar companion (Luhman 2014). A survey of nearby field stars with Hipparcos astrometric data (Perryman et al. 1997) by García-Sánchez et al. (1999) identified only a single candidate with a pass of within $0.9 \mathrm{pc}$ of the Sun (Gl 710; $1.4 \mathrm{Myr}$ in the future at $\sim 0.34 \mathrm{pc}$ ); however, it is predicted that $\sim 12$ stars pass within $1 \mathrm{pc}$ of the Sun every Myr (GarcíaSánchez et al. 2001). A recent analysis by Bailer-Jones (2014) of the orbits of $\sim 50,000$ stars using the revised Hipparcos astrometry from van Leeuwen (2007), identified four Hipparcos stars whose future flybys may bring them within $0.5 \mathrm{pc}$ of the Sun (however, the closest candidate HIP 85605 has large astrometric uncertainties; see discussion in Section 3).

A low-mass star in the solar vicinity in Monoceros, WISE J072003.20-084651.2 (hereafter W0720 or "Scholz's star") was recently discovered with a photometric distance of $\sim 7 \mathrm{pc}$ and initial spectral classification of M9 \pm 1 (Scholz 2014). This nearby star likely remained undiscovered for so long due to its combination of proximity to the Galactic plane $(b=+2: 3)$, optical dimness $(V=18.3 \mathrm{mag})$, and low proper motion $\left(\sim 0{ }^{\prime \prime} 1 \mathrm{yr}^{-1}\right)$. The combination of proximity and low tangential velocity for W0720 $\left(V_{\mathrm{tan}} \simeq 3 \mathrm{~km} \mathrm{~s}^{-1}\right)$ initially drew our attention to this system. If most of the star's motion was radial, it was possible that the star may have a past or future close pass to the Sun. Indeed, Burgasser et al. (2014) and Ivanov et al. (2014) have recently reported a high positive radial velocity. Burgasser et al. (2014) resolved W0720 as a M9.5+T5 binary and provided a trigonometric parllax distance of $6.0_{-0.9}^{+1.2} \mathrm{pc}$. Here we investigate the trajectory of the W0720 system with respect to the solar system, and demonstrate that the star recently ( $\sim 70,000$ years ago) passed through the Oort Cloud.

\section{DATA AND ANALYSIS}

We obtained medium-resolution spectroscopy of W0720 on UT dates 2013 November 17 and 19 with the Southern African Large Telescope and Magellan telescopes, in the optical and near-infrared, respectively. As summarized in Ivanov et al. (2014), we estimated the spectral type of W0720 to be $\mathrm{LO} \pm 1$, and measured a radial velocity of $77.6 \pm$ $2.5 \mathrm{~km} \mathrm{~s}^{-1}$. Recent adaptive optics imaging and high-resolution spectroscopy by Burgasser et al. (2014) indicated that the star is actually a low-mass binary classified as M9.5+T5, with projected separation $0.8 \mathrm{AU}$, and a multi-epoch mean radial velocity of $83.1 \pm 0.4 \mathrm{~km} \mathrm{~s}^{-1}$. Given the radial velocity accuracy, number of reported epochs (11), and improved 
astrometric solution provided by the Burgasser et al. (2014) study, we simply adopt their parameters for our calculations: $\alpha=07: 20: 03.21$ (ICRS; epoch 2014.0), $\delta=-08^{\circ} 46^{\prime} 51$ " 83 (ICRS; epoch 2014.0), $\mu_{\alpha}=-40.3 \pm$ $0.2 \mathrm{mas} \mathrm{yr}^{-1}, \mu_{\delta}=-114.8 \pm 0.4 \mathrm{mas} \mathrm{yr}^{-1}, \varpi=166 \pm 28$ mas, $d=6.0_{-0.9}^{+1.2} \mathrm{pc}, v_{\text {radial }}=83.1 \pm 0.4 \mathrm{~km} \mathrm{~s}^{-1}, v_{\text {total }}=83.2$ $\pm 0.4 \mathrm{~km} \mathrm{~s}^{-1}$ (all $1 \sigma$ uncertainties).

Using the Galactic velocity ellipsoids and population normalizations from Bensby et al. (2003), we estimate that W0720 has probabilities of $77.3,22.5$, and $0.2 \%$ of belonging to the thin disk, thick disk, and halo Galactic populations, respectively. The spectral classification indices are consistent with a thin disk star of approximately solar composition (Burgasser et al. 2014; classified "dM" based on $\zeta=1.034 \pm$ 0.018; Lépine et al. 2007). Classification as a metal-poor subdwarf is ruled out, and given the correlation between metallicity and kinematic properties for M-type stars (Savcheva et al. 2014), it is unlikely that W0720 belongs to either the thick disk or halo. Among 890 nearby solar-type stars from a chromospheric activity-velocity catalog (Jenkins et al. 2011), only three have Galactic velocity components within $\pm 10 \mathrm{~km} \mathrm{~s}^{-1}$ of W0720's velocity (HIP 51500, 63851, 117499), and all three are less chromospherically active than our Sun $\left(\log R_{\mathrm{HK}}^{\prime} \leqslant-4.95\right)$ and have inferred chromospheric ages in the range 4-8 Gyr (Mamajek \& Hillenbrand 2008). W0720's velocity is similar to that of the Hercules dynamical stream, a kinematic group of stars of heterogenous ages and composition, likely perturbed to the solar circle from smaller Galactocentric radii due to dynamical interactions with the Galactic bar (Bensby et al. 2007). Based on its status as an old thin disk star, consideration of the ages of Sun-like stars of similar velocity, and the isochronal ages for other Hercules stream members (Bensby et al. 2014), we adopt an age for W0720 of 3-10 Gyr ( $2 \sigma$ range). Interpolating the mass-age estimates for the W0720 components from Burgasser et al. (2014) for solar composition, this age range maps to component masses of $M_{A}=86 \pm 2 M_{\mathrm{Jup}}$ and $M_{B}=65 \pm$ $12 M_{\text {Jup }}(2 \sigma$ uncertainties $)$. The hydrogen-burning mass limit for stars is near $\sim 75 M_{\text {Jup }}$ (Saumon \& Marley 2008; Dieterich et al. 2014), hence W0720A is probably a low-mass star, and W0720B is probably a brown dwarf.

We integrated the orbit of W0720 and the Sun with a realistic Galactic gravitational potential using the NEMO Stellar Dynamics Toolbox (Teuben 1995; Barenfeld et al. 2013). With the current velocity data, we simulated $10^{4}$ orbits of W0720 and the Sun, sampling the observed astrometric values and observational uncertainties for W0720 using Gaussian deviates. From these $10^{4}$ simulations which take into account the observational uncertainties, we find that W0720 passed as close as $\Delta=0.252_{-0.068}^{+0.111} \mathrm{pc}\left(1 \sigma ;{ }_{-0.110}^{+0.317} \mathrm{pc}\right.$ $2 \sigma)$ or $52.0_{-13.9}^{+22.9} \mathrm{kAU}\left(1 \sigma ;{ }_{-22.7}^{+65.5} \mathrm{kAU} 2 \sigma\right)$ of the Sun. The time of closest approach was $70.4_{-9.8}^{+14.7}\left(1 \sigma{ }_{-17.6}^{+36.2} 2 \sigma\right)$ kya ${ }^{9}$. Figure 1 shows the distributions of the closest approach separations and times for W0720 and the Sun for the $10^{4}$ orbit simulations. The median nearest pass position from the simulations was $(X, Y$, $Z)=(-0.122,+0.120,+0.185)$ pc (here quoted in a comoving frame centered on the solar system barycenter, where $X$ is in the current direction of the Galactic center, $Y$ is in the current direction of Galactic rotation, and $Z$ is toward the current north Galactic pole), with approximate $1 \sigma$ uncertainties of $\left(\sigma_{X}, \sigma_{Y}\right.$,

\footnotetext{
kya $=$ thousand years ago.
}

$\left.\sigma_{Z}\right)=(0.047,0.045,0.068) \mathrm{pc}$, corresponding to celestial position $\left(\alpha_{\text {ICRS }}, \delta_{\text {ICRS }} \simeq 170^{\circ} \pm 29^{\circ},+68^{\circ} \pm 14^{\circ} ; 1 \sigma\right.$ unc. $)$, in the vicinity of Ursa Major. As a check, we also calculated a linear trajectory that ignores the Galactic potential, finding that W0720's closest approach was $70.7 \mathrm{kya}$ at 0.25 parsec at a barycentric position in Galactic coordinates of $(X, Y$, $Z=-0.125,+0.119,+0.185 \mathrm{pc})$. Note that given the short timespan, the predictions of the time and position of the W0720-Sun minimum pass from the linear trajectory agrees with the more accurate orbit integration to better than $2.5 \%$. At its closest pass W0720 would have had proper motion exceeding any known star: $70^{\prime \prime} \mathrm{yr}^{-1}$ (i.e., capable of traversing a full Moon in $26 \mathrm{yr}$; cf. the current highest proper motion star, Barnard's star, with 10 !'73 $\mathrm{yr}^{-1}$ ).

The predicted Galactic coordinates of the nearest pass of the binary system $\left(\ell, b=135^{\circ} \pm 15^{\circ}, 47^{\circ} \pm 13^{\circ}\right)$ is near one of the two strong peaks in the longitude of aphelia distribution of new class I comets from the Oort Cloud $\left(\ell=135^{\circ} \pm 15^{\circ}\right)$ (Matese et al. 1999). However, this appears to be coincidence, as any comets on eccentricity $\sim 1$ orbits from the vicinity of the nearest pass $\sim 70$ kya would have periods of $\sim 4 \mathrm{Myr}$, and hence require $\sim 2 \mathrm{Myr}$ to reach the inner solar system. Also, the two primary peaks in the distribution of longitudes of aphelia for long-period comets are reasonably explained by the effects of the solar motion and Galactic tide (Feng \& Bailer-Jones 2014). Among $10^{4}$ simulations, the nearest past separation between W0720 and the Sun was $\Delta=0.087 \mathrm{pc}(18.0 \mathrm{kAU})$, and this was the only simulation that brought W0720 within the classical boundary of the dynamically active inner Oort Cloud ("Hills Cloud"; Hills 1981; Weissman 1996) at $a<20 \mathrm{kAU}$. Approximately $79 \%$ of the simulations brought W0720 within $0.337 \mathrm{pc}$ of the Sun, the previously closest estimated pass of a known star to the solar system (Gliese $710 ; 0.337$ pc, $1.4 \mathrm{Myr}$ in the future; García-Sánchez et al. 2001). Approximately $99.96 \%$ of the simulated trajectories brought the star within the Sun's tidal radius of 1.35 pc (Mamajek et al. 2013), and $98 \%$ of the simulated trajectories brought the star within the maximal range of semimajor axes for retrograde orbiting Oort Cloud comets ( 120 kAU; García-Sánchez et al. 1999). The rarity of stellar passes with such a small impact parameter can be assessed from the analysis of García-Sánchez et al. (2001). Extrapolating the power-law distribution of minimum separations versus cumulative number of encounters per Myr from García-Sánchez et al. (2001), one estimates that encounters by stellar systems within $0.25 \mathrm{pc}$ of the Sun occur with a frequency of $0.11 \mathrm{Myr}^{-1}$ or once every $\sim 9.2 \mathrm{Myr}$. For comparison, flybys this close $(0.25 \mathrm{pc})$ are statistically rare $(\sim 2.4 \%)$ among encounters by all stellar systems that penetrate the Sun's tidal radius of $\sim 1.35$ pc (Mamajek et al. 2013), of which 4.5 occur per Myr (García-Sánchez et al. 2001).

The flyby's gravitational interaction is in the regime where the Sun's influence on the star's trajectory (and vice versa) is negligible (Collins \& Sari 2010): $G M_{\odot} / b v_{*}^{2} \simeq 10^{-5.6} \ll 1$, where $G$ is the Newtonian constant, $M_{\odot}$ is the Sun's mass, $b$ is impact parameter (minimum Sun-star separation), and $v_{*}$ is the velocity of the star with respect to the solar system barycenter. Major comet showers, where the flux of long-period comets increases by factors of $>10$ are likely limited to cases of high mass interlopers passing within $\sim 10 \mathrm{kAU}$ of the Sun (Heisler $\&$ Tremaine 1986), and are exceedingly rare $\left(<10^{-3} \mathrm{Myr}^{-1}\right.$; extrapolating from results by García-Sánchez et al. 2001). A perturbed comet with new aphelion similar to that of the Sun- 


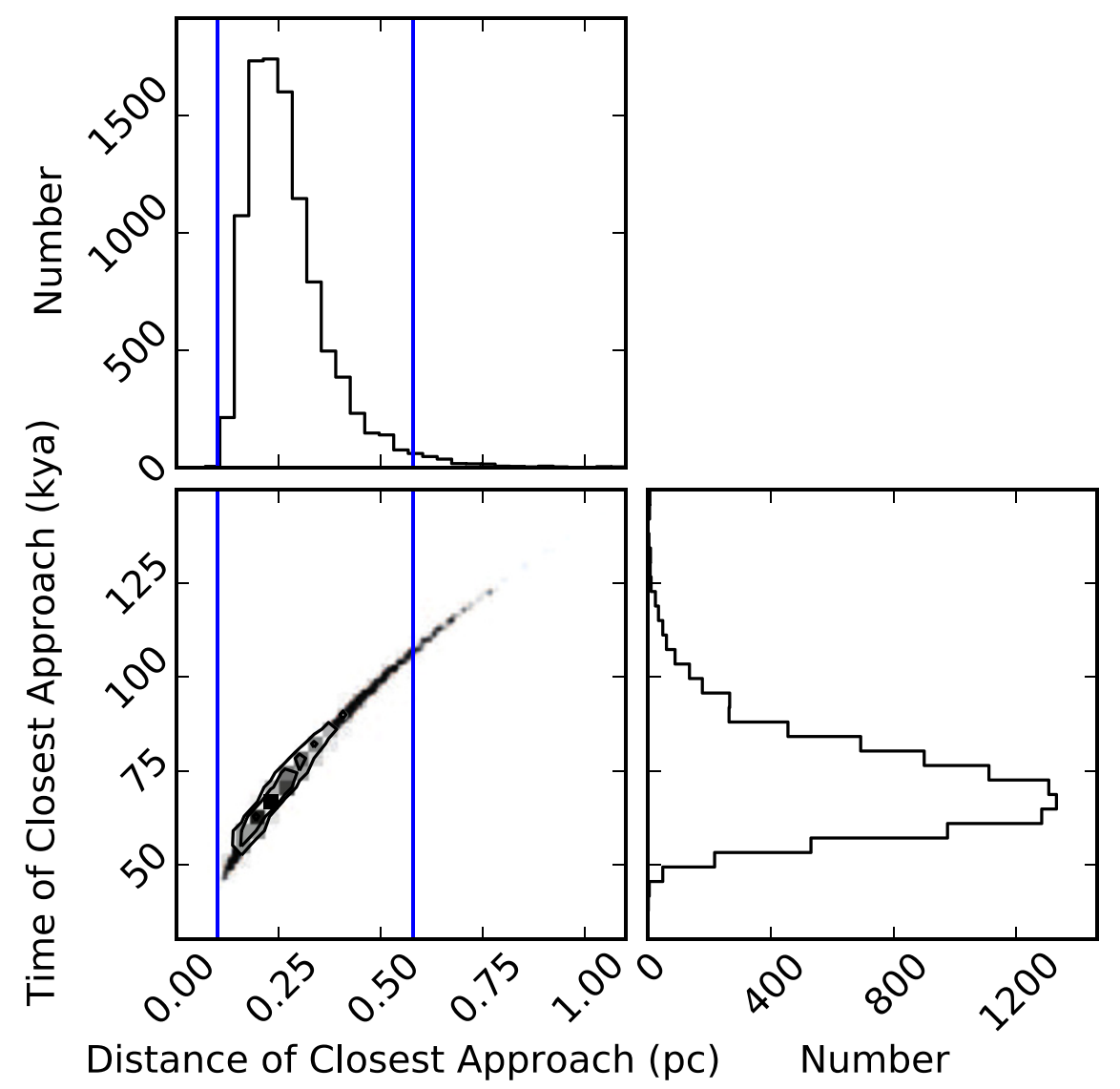

Figure 1. Lower left: density plot of the distribution of minimum separations between W0720 and the Sun (in pc) versus time of minimum separation (in thousands of years ago; "kya") for $10^{4}$ Galactic orbit simulations. The maximum semimajor axis for retrograde orbiting Oort Cloud comets is $\sim 0.58 \mathrm{pc}$ (right blue solid line), and the dynamically active inner Oort Cloud is at $<0.10 \mathrm{pc}$ (left blue solid line). The entire region plotted is within the Sun's tidal radius $(\sim 1.35 \mathrm{pc}$ ). Upper left: histogram of simulated closest W0720-Sun separations (pc). Lower right: histogram of simulated times of closest W0720-Sun separations (kya).

W0720 minimum separation, but with perihelion in the planetary region of the solar system, will have $a \simeq 26 \mathrm{kAU}$ and $P \sim 4.2$ Myr. Although comets throughout the Oort cloud may be perturbed by an interloper star onto trajectories that would bring them to the inner solar system (Weissman 1996), the highest density of outer Oort cloud comets should exist at smaller orbital distances (Heisler 1990), so most of the perturbed comets should originate from the vicinity of the star's closest pass to the Sun. Hence, any enhancement on the long-period comet flux should become manifest $2 \mathrm{Myr}$ in the future. A proxy indicator of the encounter-induced flux of Oort Cloud comets is defined as $\gamma=M_{*} / \nu_{*} b$ (Feng \& BailerJones 2014 using the variables as defined earlier, and with $M_{*}$ being the mass of the interloper binary W0720). Using the $10^{4}$ simulations, we estimate that the W0720 flyby induced $\gamma \sim$ $10^{-7.48 \pm 0.15(1 \sigma)} M_{\odot} \mathrm{km}^{-1} \mathrm{~s} \mathrm{AU}^{-1}$. Simulations by Feng \& Bailer-Jones (2014) suggest that encounters with $\gamma<10^{-5.3}$ are unlikely to generate an enhancement in the distribution of longitudes for long-period comets compared to that predicted to be generated by Galactic tidal effects. All of the $10^{4}$ simulated orbits had $\gamma<10^{-7.0}$, hence the pass of the W0720 system should have a negligible statistical impact on the flux of longperiod comets during the coming millenia.

\section{THE CLOSEST KNOWN FLYBY?}

The proximity of W0720's flyby to the solar system can be compared to those of Hipparcos stars recently studied by
Bailer-Jones (2014). He identified one star (HIP 85605) with a flyby closer than the median minimum separation that we estimated for W0720 (0.25 pc); however, two future flybys (HIP 89825 [G1 710] and HIP 63721) both have $\Delta \simeq 0.27 \mathrm{pc}$ $\left(0.27_{-0.17}^{+0.17}\right.$ pc and $0.27_{-0.23}^{+0.40} \mathrm{pc}$, respectively; $\left.90 \% \mathrm{CL}\right)$. BailerJones (2014) predicts that HIP 85605 will pass within $\Delta=0.10_{-0.06}^{+0.10} \mathrm{pc}(90 \% \mathrm{CL})$ of the Sun $\sim 332 \mathrm{kyr}$ in the future. Unfortunately, as Bailer-Jones (2014) points out, HIP 85605 is a visual binary, and there is considerable dispersion in its published proper motions and its Hipparcos parallax is of low accuracy. The solution which brings HIP 85605 close to the Sun relies on the Tycho- 2 proper motion $\left(\mu_{\alpha}, \mu_{\delta}=+4.0\right.$, $-7.6 \pm 2.0,1.9 \mathrm{mas} \mathrm{yr}^{-1}$; $\mathrm{H} \varnothing \mathrm{g}$ et al. 2000), the revised Hipparcos parallax of $\varpi=146.84 \pm 29.81 \mathrm{mas}$, and the Pulkovo radial velocity of $-21.0 \pm 0.3 \mathrm{~km} \mathrm{~s}^{-1}$ (Gontcharov 2006 as reported in the XHIP compiled catalog; Anderson $\&$ Francis 2012). If any of these values are substantially in error, then the proximity of the flyby solution is likely to be spurious. Both the original and revised Hipparcos astrometric solutions (Perryman et al. 1997; van Leeuwen 2007) had much larger proper motions than the Tycho-2 solution. Bailer-Jones (2014) mentions that a reanalysis of the Hipparcos astrometric solution by F. van Leeuwen suggests "that the Hipparcos-2 parallax of HIP 85605 and its (relatively large) uncertainty are valid, but that on account of the large residuals and the complex nature of this system the solution should be treated with caution." 
Unfortunately, the Hipparcos parallax for HIP 85605 leads to some astrophysical inconsistency. As Bailer-Jones (2014) note, HIP 85605's B-V color (1.1 mag) is consistent with a Ktype star, whereas the absolute magnitude calculated using its revised Hipparcos parallax $\left(M_{V}=11.86 \pm 0.45 \mathrm{mag}\right)$ is more consistent with an M dwarf. We find that HIP 85605's colors from Hipparcos and 2MASS $\left(B-V=1.10 \pm 0.11, V-K_{s}\right.$ $\simeq 2.66 \pm 0.07, J-H \simeq 0.53 \pm 0.03, H-K_{s} \simeq 0.12 \pm$ 0.03 ) are all consistent with a $\sim \mathrm{K} 4$ dwarf (Pecaut \& Mamajek 2013), in agreement with the spectral template fitting analysis of Pickles \& Depagne (2010; which also yielded $\mathrm{K} 4 \mathrm{~V})$. D. Latham (2015, private communication) has visually confirmed that five spectra of HIP 85605 taken with the CfA Digital Speedometer (as reported in García-Sánchez et al. 1999) are indeed consistent with a typical K dwarf. As HIP 85605 is spectrophotometrically a K dwarf, the Hipparcos -derived absolute magnitude $\left(M_{V} \simeq 11.9\right)$ places the star nearly five magnitudes fainter than the main sequence $\left(M_{V}^{\mathrm{MS}} \simeq\right.$ 7.0) for a star of its color-too faint to be simply a metal-poor dwarf, yet too red and luminous for a white dwarf ${ }^{10}$. Hence, most likely HIP 85605 is a $\sim \mathrm{K} 4 \mathrm{~V}$ at $d \simeq 60 \mathrm{pc}$ and the Hipparcos parallax is erroneous. At $d \simeq 60 \mathrm{pc}$, HIP 85605 has a slightly different velocity $(U, V, W=-10.7,-14.3$, $\left.-11.6 \mathrm{~km} \mathrm{~s}^{-1}\right)$ compared to that used in the simulation by Bailer-Jones (2014), and its "flyby" of the Sun correspondingly moves further into the future and further away $(\sim 2.8 \mathrm{Myr}$ in future, $\sim 10 \mathrm{pc}$ away). We conclude that HIP 85605 is unlikely to penetrate the Sun's Oort Cloud, and that W0720 now appears to have the closest flyby of any known star.

\section{DISCUSSION}

Given its current visual magnitude of $V \simeq 18.3$ (Ivanov et al. 2014), at its closest approach of $\sim 0.25 \mathrm{pc}$, Scholz's star (W0720) would have had an apparent magnitude of $V \simeq 10.3$, brighter than the current nearest star (Proxima; $V=11.2$ ) but still much dimmer than the faintest naked eye stars $(V \sim 6)$. However, W0720 is an active M dwarf star (Burgasser et al. 2014), and $V$-band flares have been observed to exceed 9 mag on timescales of minutes among such stars, and brief flares exceeding 12 mag may be possible (Schmidt et al. 2014). Flares among the coolest $\mathrm{M}$ dwarfs have been witnessed with energies of $\sim 10^{34} \mathrm{ergs}$ (Schmidt et al. 2014) and luminosities of $\sim 10^{29}-10^{30} \mathrm{erg} \mathrm{s}^{-1}$. If W0720 experienced occasional flares similar to those of the active M8 star SDSS J022116.84 + 194020.4 (Schmidt et al. 2014), then the star may have been rarely visible with the naked eye from Earth $(V<6 ; \Delta V<-4)$ for minutes or hours during the flare events. Hence, while the binary system was too dim to see with the naked eye in its quiescent state during its flyby of the solar system $\sim 70$ kya, flares by the M9.5 primary may have provided short-lived transients visible to our ancestors.

Improved astrometry for W0720 via ground-based telescopes (Dieterich et al. 2014) or Gaia (de Bruijne 2012), and further radial velocity monitoring, should help reduce the uncertainties in the flyby timing and minimum separation between Scholz's star and the solar system. Past systematic searches for stars with close flybys to the solar system have been understandably focused on the Hipparcos astrometric catalog (García-Sánchez et al. 1999; Bailer-Jones 2014); however, it contains relatively few $\mathrm{M}$ dwarfs relative to their cosmic abundance. Searches in the Gaia astrometric catalog for nearby $\mathrm{M}$ dwarfs with small proper motions and large parallaxes (i.e., with small tangential velocities) will likely yield addition candidates.

E.E.M. acknowledges support from NSF grant AST1313029. A.Y.K. and P.V. acknowledge the support from the National Research Foundation (NRF) of South Africa. We thank Dave Latham, Alice Quillen, Kevin Luhman, Cameron Bell, Dave Cameron, Peter Teuben, Segev Ben-Zvi, Adam Burgasser, Ralf-Dieter Scholz, Matt Multunas, and Richard Sarkis for discussions. We thank the referee for a very timely and useful report.

\section{REFERENCES}

Anderson, E., \& Francis, C. 2012, AstL, 38, 331

Bailer-Jones, C. A. L. 2011, MNRAS, 416, 1163

Bailer-Jones, C. A. L. 2014, A\&A, in press (arXiv:1412.3648)

Barenfeld, S. A., Bubar, E. J., Mamajek, E. E., \& Young, P. A. 2013, ApJ, 766, 6

Bensby, T., Feltzing, S., \& Lundström, I. 2003, A\&A, 410, 527

Bensby, T., Feltzing, S., \& Oey, M. S. 2014, A\&A, 562, A71

Bensby, T., Oey, M. S., Feltzing, S., \& Gustafsson, B. 2007, ApJL, 655, L89

Biermann, L., Huebner, W. F., \& Lust, R. 1983, PNAS, 80, 5151

Burgasser, A. J., Gillon, M., Melis, C., et al. 2014, AJ, in press (arXiv:1410.4288)

Collins, B. F., \& Sari, R. 2010, AJ, 140, 1306

Davis, M., Hut, P., \& Muller, R. A. 1984, Natur, 308, 715

de Bruijne, J. H. J. 2012, Ap\&SS, 341, 31

Dieterich, S. B., Henry, T. J., Jao, W.-C., et al. 2014, AJ, 147, 94

Feng, F., \& Bailer-Jones, C. A. L. 2014, MNRAS, 442, 3653

García-Sánchez, J., Preston, R. A., Jones, D. L., et al. 1999, AJ, 117, 1042

García-Sánchez, J., Weissman, P. R., Preston, R. A., et al. 2001, A\&A, 379,634

Gontcharov, G. A. 2006, AstL, 32, 759

Heisler, J. 1990, Icar, 88, 104

Heisler, J., \& Tremaine, S. 1986, Icar, 65, 13

Hills, J. G. 1981, AJ, 86, 1730

Høg, E., Fabricius, C., Makarov, V. V., et al. 2000, A\&A, 355, L27

Ivanov, V. D., Vaisanen, P., Kniazev, A. Y., et al. 2014, A\&A, in press (arXiv:1410.6792)

Jenkins, J. S., Murgas, F., Rojo, P., et al. 2011, A\&A, 531, A8

Lépine, S., Rich, R. M., \& Shara, M. M. 2007, ApJ, 669, 1235

Luhman, K. L. 2014, ApJ, 781, 4

Mamajek, E. E., Bartlett, J. L., Seifahrt, A., et al. 2013, AJ, 146, 154

Mamajek, E. E., \& Hillenbrand, L. A. 2008, ApJ, 687, 1264

Matese, J. J., Whitman, P. G., \& Whitmire, D. P. 1999, Icar, 141, 354

Oort, J. H. 1950, BAN, 11, 91

Pecaut, M. J., \& Mamajek, E. E. 2013, ApJS, 208, 9

Perryman, M. A. C., Lindegren, L., Kovalevsky, J., et al. 1997, A\&A, 323, L49

Pickles, A., \& Depagne, É 2010, PASP, 122, 1437

Rickman, H. 2014, M\&PS, 49, 8

Saumon, D., \& Marley, M. S. 2008, ApJ, 689, 1327

Savcheva, A. S., West, A. A., \& Bochanski, J. J. 2014, ApJ, 794, 145

Schmidt, S. J., Prieto, J. L., Stanek, K. Z., et al. 2014, ApJL, 781, L24

Scholz, R.-D. 2014, A\&A, 561, A113

Teuben, P. 1995, in ASP Conf. Ser. 77, Astronomical Data Analysis Software and Systems IV, ed. R. A. Shaw, H. E. Payne, \& J. J. E. Hayes (San Francisco, CA: ASP), 398

van Leeuwen, F. 2007, Astrophys. Space Sci. Libr., 350

Weissman, P. R. 1996, in ASP Conf. Ser. 107, Completing the Inventory of the Solar System, ed. T. W. Rettig, \& J. M. Hahn (San Francisco, CA: ASP), 265

Whitmire, D. P., \& Jackson, A. A. 1984, Natur, 308, 713

\footnotetext{
${ }^{10}$ See RECONS HR diagram for $10 \mathrm{pc}$ sample (http://recons.org/hrd.2010.0. html) and http://dx.doi.org/10.6084/m9.figshare.1284334.
} 